\title{
Metastable pin fin boiling
}

\author{
W.W. Lin, J.C. Yang, D.J. Lee* \\ Department of Chemical Engineering, National Taiwan University, Taipei 10617, Taiwan
}

Received 25 January 1999; received in revised form 28 June 1999

\begin{abstract}
This work has, for the first time, numerically and experimentally proved the existence of metastable regimes in fin boiling process. The linearly stable steady states were subject to external perturbations. As the linearly stable steady state moves toward the critical points on the boiling curve by increasing base temperature, which is linearly unstable in nature, the capability of the steady state to tolerate perturbation becomes weaker. Stable and metastable regimes could be subsequently identified. To operate at metastable boiling regime might induce (nonlinear) instability if the magnitude of external perturbation is large enough. Experimental results support these numerical findings. A proper boiling fin design should take into consideration both the stable and metastable regimes. (C) 2000 Elsevier Science Ltd. All rights reserved.
\end{abstract}

\section{Introduction}

Several books had summarized investigations analyzing fins with a constant heat transfer coefficient (e.g., see Kern and Kraus [1]). When boiling occurs on a fin, the heat transfer coefficient along the fin is definitely not constant. Instigated by the pioneering works of Haley and Westwater [2] and Lai and Hsu [3], fin boiling process has received extensive attention. Kraus [4] and Liaw and Yeh [5] reviewed related literature. Both the heat transfer rate from the fin base, as well as the feasible operational temperature range, might increase markedly at compared with the case without a fin [6]

When liquid boils on a fin, complicated heat transfer configurations might appear. For instance, when the fin base temperature is located in film boiling region, at least three situations might occur on the fin surface: film boiling alone, film followed by transition boiling,

\footnotetext{
* Corresponding author. Tel.: +886-2-2362-5230; fax: $+886-2-2362-3040$

E-mail address: djlee@ccms.ntu.edu.tw (D.J. Lee).
}

and film + transition + nucleate boiling. (Obviously, the material's melting point must be higher than the maximum temperature considered here for the boiling to be possible.) By assuming a power-law type temperature dependence of the heat transfer coefficient for each boiling mode, the steady-state temperature distribution along the fin and the base heat flow for various boiling configurations can be found. Fin effectiveness and fin efficiency can then be evaluated from these data and, subsequently, the fin design information is obtained $[7,8]$. A prerequisite for using of these steady-state solutions in practice is that they are stable to external perturbations.

Liaw and Yeh [5] analyzed the stability characteristics for only one boiling mode on the fin. Lin and Lee [9] had provided a first detailed stability analysis on the steady-state solution for multi-mode boiling on a straight pin fin. They demonstrated that when film and transition boiling coexist on the fin surface, or only the transition boiling has covered the entire fin, the operation is stable only if the fin length is less than some critical value. When transition and nucleate boiling coexist on a fin, or in 


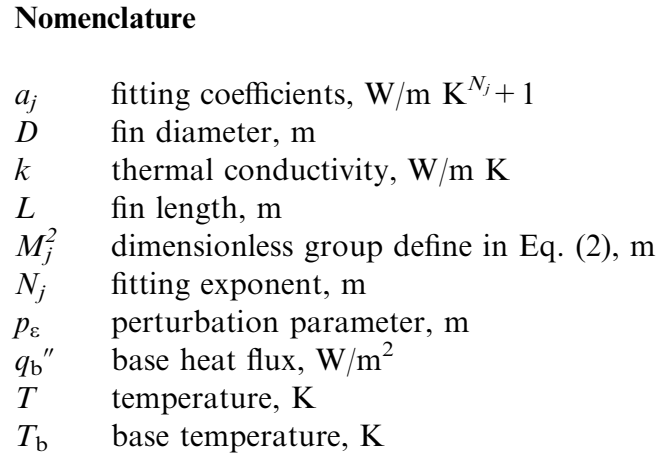

$\begin{array}{ll}\Delta T_{\mathrm{b}} & \text { base superheat }\left(T_{\mathrm{b}}-T_{\mathrm{sat}}\right), \mathrm{K} \\ T_{\text {sat }} & \text { saturation temperature, } \mathrm{K} \\ t & \text { time, } \mathrm{s} \\ X & \text { dimensionless position, } \mathrm{m} \\ x & \text { axial position, } \mathrm{m} \\ \alpha & \text { thermal diffusivity, } \mathrm{m}^{2} / \mathrm{s} \\ \tau & \text { dimensionless time, } \mathrm{m}^{2} / \mathrm{s} \\ \Theta & \text { dimensionless temperature, } \mathrm{m}^{2} / \mathrm{s} \\ \bar{\Theta} & \text { dimensionless perturbation temperature, } \mathrm{m}^{2} / \mathrm{s} \\ \Theta^{\mathrm{S}} & \text { dimensionless steady-state temperature, } \mathrm{m}^{2} / \mathrm{s}\end{array}$

the three-mode boiling (film + transition + nucleate boiling), the entering of nucleate boiling at the fin tip stabilizes the boiling process. A follow-up study experimentally proved their theoretical prediction [10]. The critical points of multi-mode boiling on a fin, whose role resembles the $\mathrm{CHF}$ and $\mathrm{MHF}$ points in pool boiling, are available [11,12].

The points separating stable and metastable regimes on the boiling curve are essential for practical applications. Critical points such as the critical heat flux (CHF) and minimum heat flux (MHF) points indicate the absolutely unstable points at which the system cannot tolerate an infinitesimal disturbance. On the other hand, a heater in metastable nucleate boiling mode can tolerate a finitemagnitude disturbance to burnout. A dry patch of a sufficiently large size can push the heater to film boiling. Such an occurrence is termed 'nonhydrodynamic burnout' [13]. In contrast, if the heater is in a stable nucleate boiling mode, no transit to film boiling occurs regardless of the magnitude of dry patch. Similar observations can be applied to film boiling curve as well [14]. Identifying the points separating stable and metastable boiling regimes is a pertinent task in boiler design and operation. Lin and Lee [15-17] for the first time experimentally identified the points separating stable and metastable boiling modes on an indirectly conductive heating surface. Analogy with the wire boiling system is established [18].

For pin fin boiling, within certain operational conditions, the so-called USS (upper steady state), representing the state of high heat transfer efficiency, and the LSS (lower steady state), representing those of a low heat transfer efficiency, can co-exist $[9,10]$. Although the criteria of the critical points of USS and LSS are now available, their relative stability characteristics remain unclear. According to the wire boiling or plate boiling experiments in literature, wondering arises naturally whether there exists metastable regime in fin boiling system as well. If such a speculation is valid, a fin should be designed not only below the critical point, but also within the stable regime of USS, to prevent the possible burnout induced by external perturbation (dry patch). If there does exist metastable regime in fin boiling, then, along USS, the linearly stable steady state would become less stable with increasing base temperature. Restated, when subject to the same external perturbation, the state on USS would not lose stability at lower base temperature, but would transit to LSS when base temperature becomes high enough. Similar arguments should be applicable to the steady states on LSS as well. This work examines, for the first time, the existence of metastable regime in pin fin boiling. The nonlinear analysis for governing equation of fin boiling was first conducted through numerical simulations. Next, experiments were performed for verifying the theoretical findings.

\section{Numerical simulation}

We herein numerically elucidate the effects of adding large external perturbations on the linearly stable steady-state solutions of the governing equation.

By assuming a constant thermal conductivity of the fin, the dimensionless governing equation with powerlaw type heat transfer coefficient reads [9]:

$\frac{\partial \Theta_{j}}{\partial \tau}=\frac{\partial^{2} \Theta_{j}}{\partial X^{2}}-M_{j}^{2} \Theta_{j}^{N_{j}+1}$,

where

$\Theta_{j}=\frac{T_{j}-T_{\mathrm{sat}}}{T_{b}-T_{\mathrm{sat}}}$,

$X=\frac{x}{L}$,

$\tau=\frac{\alpha t}{L^{2}}$ 
$M_{j}^{2}=\left(\frac{4 a_{j} L^{2}\left(T_{b}-T_{\mathrm{sat}}\right)^{N_{j}}}{k D}\right)$.

The two boundary conditions at the origin and the tip are

$\Theta_{i}(0)=1$,

$\frac{\mathrm{d} \Theta_{k}(1)}{\mathrm{d} X}=0$.

Other boundary conditions appear when multi-mode boiling occurs, including the continuation of temperature and heat flux at the interfaces between different modes. The index system is the same as that employed in [9]. The corresponding steady-state temperature distributions of Eq. (1), $\Theta^{\mathrm{S}}$, can be found in [7] and are not repeated here for brevity's sake. The methanol boiling data used in [19] are used for sample calculations. Notably, the $N_{j}$ in Eq. (1) are $>-1$ for film and nucleate boiling, or $<-1$ for transition boiling.

To impose a perturbation of $\bar{\Theta}$ onto the steady state $\Theta^{\mathrm{S}}$ as $\Theta=\Theta^{\mathrm{S}}+\bar{\Theta}$. If the effect of the perturbation $\bar{\Theta}$ would decay, then the steady state $\Theta^{\mathrm{S}}$ is stable to this specific perturbation. Otherwise, the steady state $\Theta^{\mathrm{S}}$ is unstable to $\bar{\Theta}$. There are infinitely many possibilities of $\bar{\Theta}$, therefore being impractical to examine them all. For fitting the requirement of boundary conditions, we herein set $\bar{\Theta}=p \varepsilon X \sin (a X)$, where $a=2.202876$. Notably, the $p_{\varepsilon}$ controls the magnitude of external perturbations. Identifying a linearly unstable state requires an infinitesimal $p_{\varepsilon}$. For identifying the regime exhibiting metastability on fin boiling, however, a finite $p_{\varepsilon}$ is needed. The resulting perturbed equation would be highly nonlinear that requires numerical solutions. We herein employed IMSL package MOLCH to finding

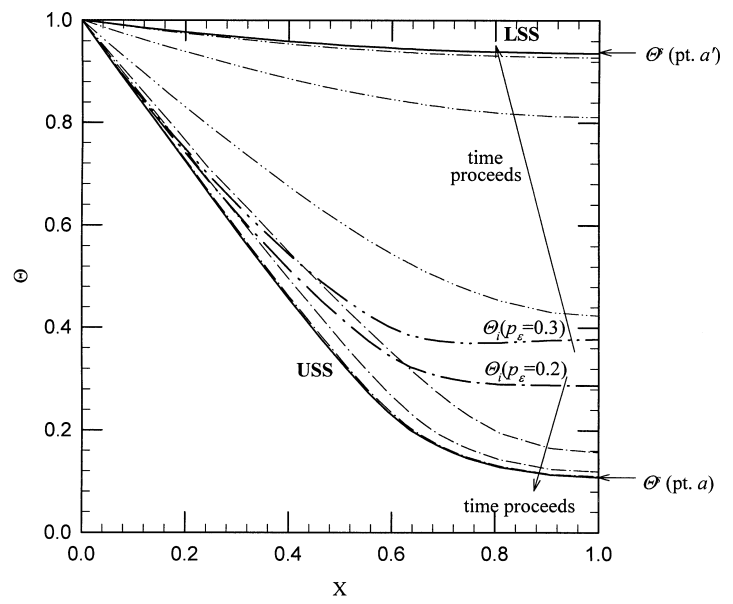

Fig. 1. Temperature evolutions in fin subject to perturbation. $\Delta T_{\mathrm{b}}=95 \mathrm{~K}, p_{\varepsilon}=0.2$ or 0.3 .

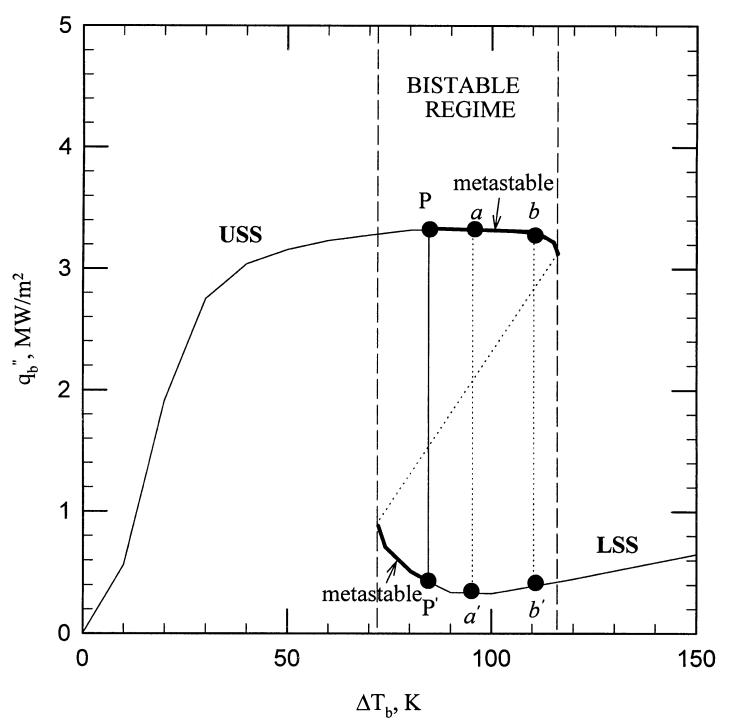

Fig. 2. Stability regimes on pin fin boiling curve. $\mathrm{PP}^{\prime}$ denotes the curve of neutral stability.

the transient solutions of perturbed equation. The maximum relative error is set as $10^{-6}$.

Figure 1 depicts the typical transients of the steadystate solution subject to perturbations. The bold curves (USS and LSS) in Fig. 1 denote the two steady-state temperature distributions for a copper fin boiling in methanol at a base superheat of $95 \mathrm{~K}$. First consider the steady-state temperature distribution of USS where the boiling state is under FTN mode, or point $a$ in Fig. 2. If the steady state is subject a perturbation of $p_{\varepsilon}=0.2$, then the transient would go back to the original steady state (follows the indication of arrow). The steady state $a$ is (nonlinearly) stable to such a perturbation with $p_{\varepsilon}=0.2$. If $p_{\varepsilon}=0.3$, on the other hand, the transient would shift to another steady state (bold curve of LSS; point $a^{\prime}$ on LSS branch of Fig. 2) rather than going back to the original steady state $a$. The steady state $a$ is hence (nonlinearly) unstable to the perturbation with $p_{\varepsilon}=0.3$. A critical $p_{\varepsilon}$ thereby exists between $0.2-0.3$ at which the two steady states, $a$ and $a^{\prime}$, are equally stable, whose value defined as $\left|p_{\varepsilon}\right|_{\text {crit }}$. If the base superheat has been raised to $110 \mathrm{~K}$, then the $\left|p_{\varepsilon}\right|_{\text {crit }}$ ranges $0.1-0.2$. In accordance with Fig. 2, such an observation correlates with the speculation that the (linearly stable) steady state on USS loses its (nonlinear) stability when the base temperature increases (moving toward the critical point).

Along LSS, on the other hand, at a base superheat of $110 \mathrm{~K}$ (point $b^{\prime}$ in Fig. 2), the transient would recede back to point $b^{\prime}$ for $p_{\varepsilon}>-0.8$. At $p_{\varepsilon}<-0.8$, the transient would move to point $b$ on USS. Its $\left|p_{\varepsilon}\right|_{\text {crit }}$ ranges $0.8-0.9$. At a base superheat of $95 \mathrm{~K}$, the critical $p_{\varepsilon}$ ranges between -0.7 and -0.8 . Or $\left|p_{\varepsilon}\right|_{\text {crit }}$ ranges 
between 0.7 and 0.8 . Restated, the (linearly stable) steady state on LSS loses its (nonlinear) stability when the base superheat decreases (moving toward the critical point as well).

Figure 3 depicts the $\left|p_{\varepsilon}\right|_{\text {crit }}$ along USS and LSS. Notably, at a base superheat less than $80 \mathrm{~K}$, no transition from USS to LSS is possible regardless of the $\left|p_{\varepsilon}\right|$ applied. Similarly, no transition from LSS to USS is possible at a base superheat greater than $120 \mathrm{~K}$. In between $(80-120 \mathrm{~K})$ there is a bistable regime where two $\left|p_{\varepsilon}\right|_{\text {crit }}$ s exist for USS and LSS, respectively. As expected, at a higher base superheat, $\left|p_{\varepsilon}\right|_{\text {crit }}$ increases along the LSS but decreases along the USS. At a base superheat of $85 \mathrm{~K}$, we note that $\left|p_{\varepsilon}\right|_{\text {crit }}$ for both branches are equal, indicating the point of neutral stability. Such a point denotes a vertical line $\mathrm{PP}^{\prime}$ in Fig. 2, which differentiates the stable and metastable regimes in pin fin boiling. The stability characteristics of the fin boiling system are hence similar to those of the plate boiling system.

The above-mentioned theoretical finding does suggest the existence of metastable regimes along both the USS as well as the LSS curves. We conducted experiments in the following sections to verify these findings.

\section{Experimental verification}

\subsection{Experimental}

Figure 4 represents the schematic of the experimental set-up, a modified version of Lin and Lee [10]. The

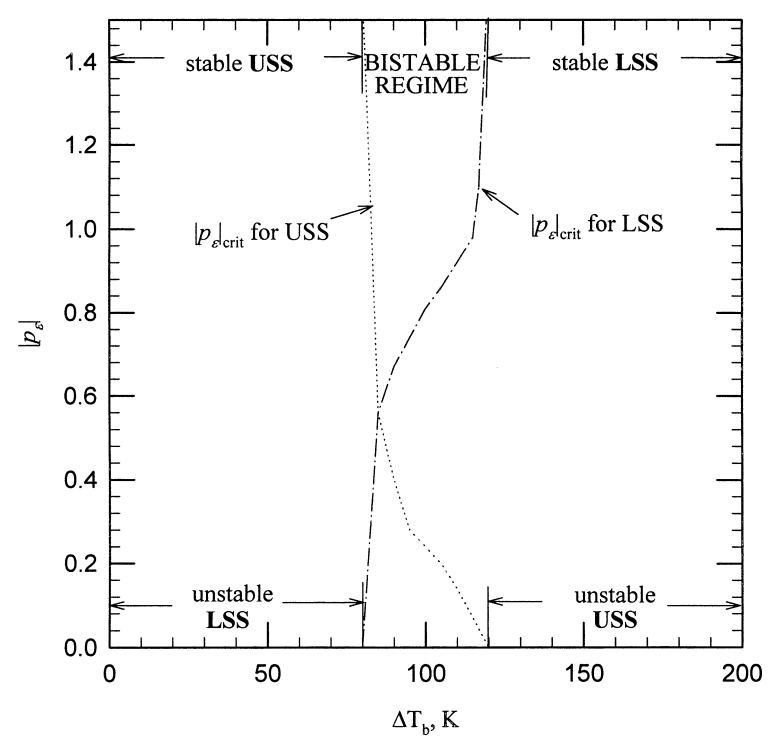

Fig. 3. Critical $\left|p_{\varepsilon}\right|$ versus fin base temperature.

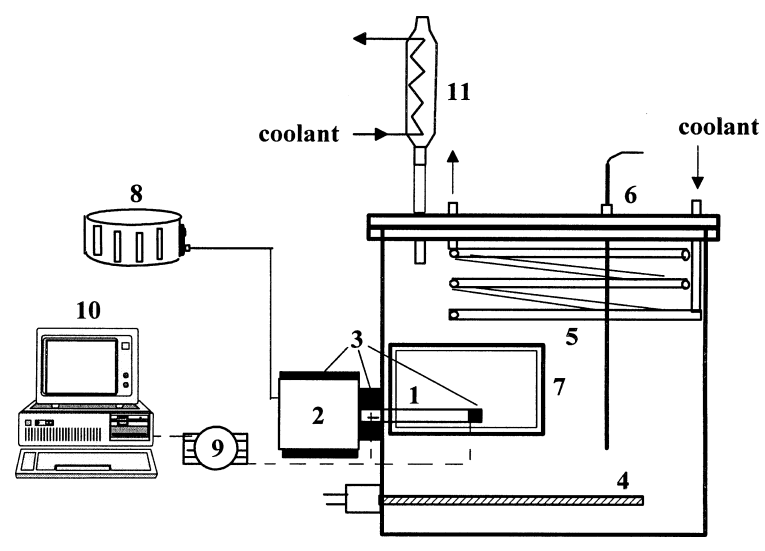

Fig. 4. Experimental set-up. (1) Fin; (2) bottom heating block; (3) insulation; (4) preheater; (5) cooling coil; (6) thermocouple; (7) view glasses; (8) transformer; (9) data acquisition system; (10) personal computer; (11) condenser.

operation pressure is atmospheric and the working liquid is methanol (purity $99 \%$ ). The testing chamber is $300(L) \times 300(W) \times 300(H) \mathrm{mm}$ in dimension and has front and back view glasses (7) for observation. A pure copper fin (1) was heated by the bottom cartridge heating block (2) whose energy is supplied by a transformer (8). In the lower section of this chamber, a preheater (4), $1.2 \mathrm{~kW}$ in capacity, was installed. In the bottom heating block, which supplies energy to the fin body, there were four cartridge heaters, each of $500 \mathrm{~W}$ capacity, giving out a maximum of $2 \mathrm{~kW}$ capacity. Temperatures at four axial positions in the heating block and at the fin tip were measured by thermocouples at a rate of $1 \mathrm{~Hz}$, sending automatically to the data acquisition system.

The fin, made of pure copper, is attached to the bottom heating block. Since the bottom heating block is well insulated, most joule heat from the bottom cartridge heaters would thereby transit into the fin bottom surface. The fin diameter is fixed as $5 \mathrm{~mm}$. The fin length under investigation is $1.0 \mathrm{~cm}$, giving an aspect ratio $(L / D)$ of 2 .

Experimental procedures were as follows. First the liquid temperature was adjusted to the desired value. After achieving the steady state condition, set on a prescribed voltage to the transformer to heat up the bottom heating block. The fin then transfers heat to the surrounding liquid via boiling. The fin bottom heat flux and superheat temperature can be estimated by extrapolating the temperature readings from the four thermocouples in the heating block. After reaching steady state condition, external perturbation was imposed. When the steady state is along the USS, where the base heat flux is high with fin tip locating at nucleate boiling mode, the temperature distribution is perturbed by drainage of pool liquid to produce dry 
patch on the fin. The liquid was then pulled back after $10 \mathrm{~s}$. If the system can return back to its original state (USS), then the operation is stable to this perturbation. Otherwise, it is unstable to this perturbation. When the state is along LSS, on the other hand, where the base heat flux is low and the whole fin is under high temperature regime, external perturbation was imposed by jetting pool liquid from the bottom the fin body. The jet can last for different period of time, referring different amplitudes of perturbation. If the system can recover its original state, the system is stable to the perturbation induced by liquid jetting. If not, the system becomes unstable to this perturbation.

Repeated experiments show that the reproducibility is satisfactory. The data acquisition system included three 8-channel thermocouple input modules (ADAM 4018, Advantech Co., Taiwan) and an isolated RS232/ RS485 converter (ADAM 4520, Advantech Co., Taiwan), whose bias error is $0.1 \%$ provided by the manufacturer, and an RS232 interface connected to a personal computer. Uncertainties having occurred are attributed primarily to the thermocouple calibration, which is, at most, $1 \mathrm{~K}$ in this study, as well as the employment of thermal conductivity data, which is estimated to not exceed $2 \%$. The uncertainties thereby existing in the extrapolated heater base temperature and the associated heat flux are estimated as 6 and $4 \%$, respectively.

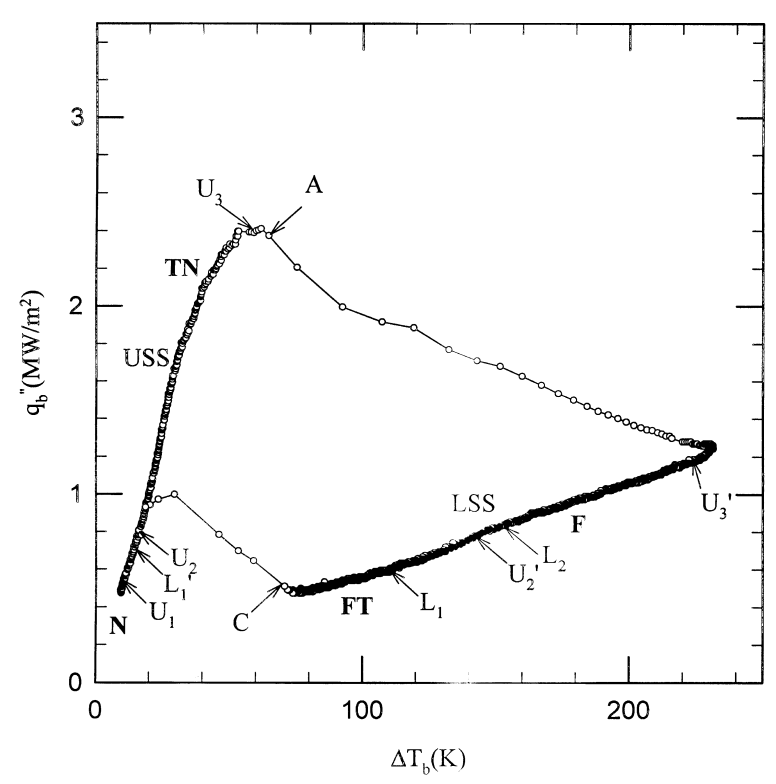

Fig. 5. Fin base heat flux versus base superheat plot. $D=5$ $\mathrm{mm}, L=10 \mathrm{~mm}$. Saturated methanol. N: nucleate boiling mode; T: transition boiling mode; F: film boiling mode.

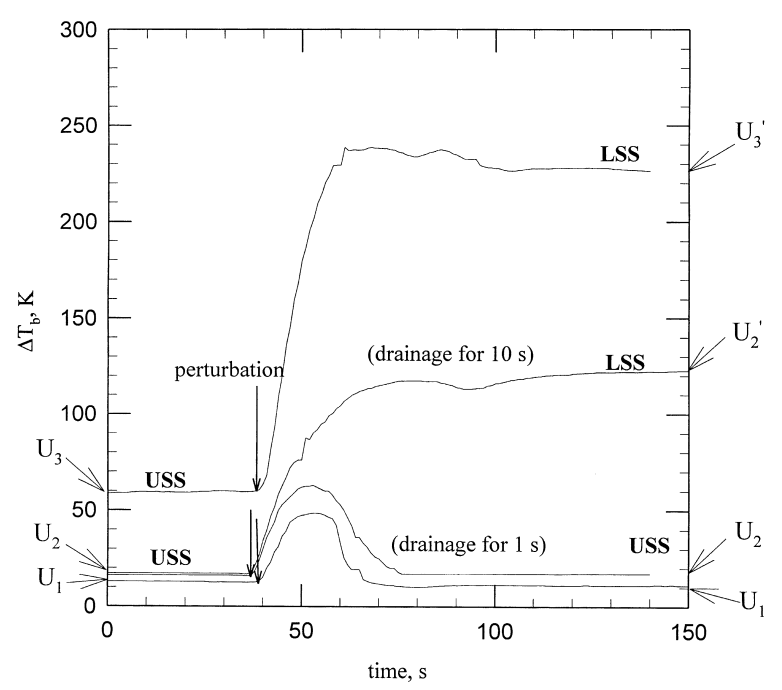

Fig. 6. Base temperature evolutions when subject to perturbations. USS regime.

\subsection{Steady-state fin boiling curve}

Figure 5 depicts the steady-state boiling curve, which is composed of the extrapolated base heat flux versus base superheat temperature data. Notably, the fin base temperature and tip temperature can easily identify the multi-mode boiling mode. The symbols $\mathrm{N}$, TN, F and FT denote respectively the nucleate boiling, transition + nucleate boiling, film boiling, and film + transition boiling mode, respectively, on the fin surface. The critical temperature corresponding to USS is $60 \mathrm{~K}$ (point A), and to LSS, $72 \mathrm{~K}$ (point C). At point A or $\mathrm{C}$ the state is linearly unstable while transition to the other steady-state branch must occur. Along the USS or the LSS curves, the operation can steadily exist, whose states are thereby linearly stable to infinitesimal perturbations.

\subsection{System response to perturbations}

Figures 6 or 7 depict the base temperature evolutions when states on USS or LSS are subject to perturbations. Consider first the USS case. For the original state $U_{1}$ in Fig. 6 (also depicted in Fig. 5), the drainage of liquid would cause the fin temperature to raise (occurrence of dry patch), but after the replenishment of liquid, the state recovers its original state. As a result, state $U_{1}$ is stable to such a perturbation. Move to a higher base temperature, $\mathrm{U}_{2}$, the state transits to LSS, $\mathrm{U}_{2}^{\prime}$ in Fig. 5, after perturbation, indicating that the state $U_{2}$ is unstable to the perturbation. Similar observation is noted for $\mathrm{U}_{3}$, which is close to the critical point A. The final steady state is on LSS, $\mathrm{U}_{3}^{\prime}$. 


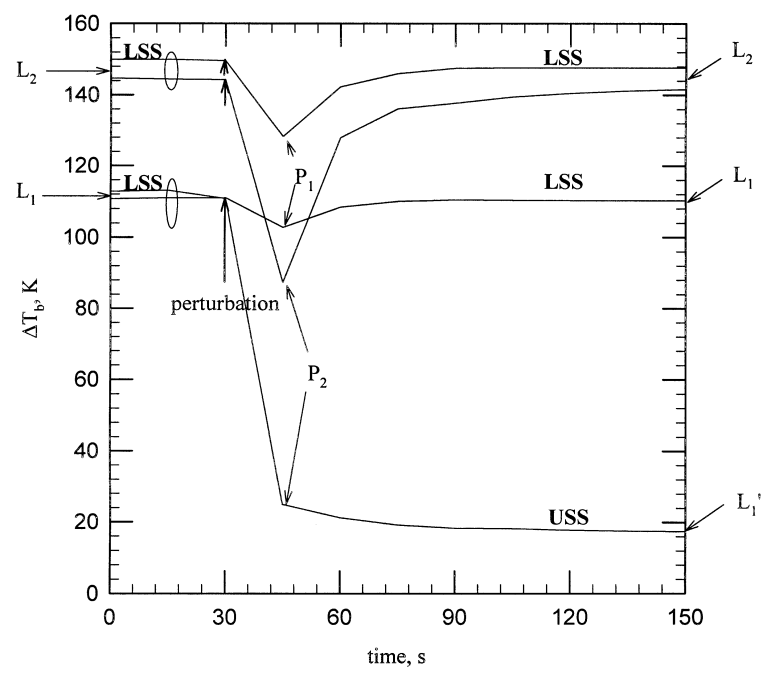

Fig. 7. Base temperature evolutions when subject to perturbations. LSS regime.

As a result, along the steady-state USS curve, the state with greater base temperature is less stable to the imposition of dry patch.

The above mentioned experimental results were subject to the same external perturbation, that is, drainage of liquid for $10 \mathrm{~s}$. To confirm whether the transition from USS to LSS (for example, from state $\mathrm{U}_{2}$ to $\mathrm{U}_{2}^{\prime}$ ) is nonlinear instability induced from a metastable regime rather than linear instability induced from $\mathrm{CHF}$ point, another set of experiment subject to a small perturbation (drainage of liquid for $1 \mathrm{~s}$ ) was conducted. As Fig. 6 reveals, the curve with superscript "*, denotes its response. Notably, the state $\mathrm{U}_{2}$ subject to a small perturbation will return back its original state. The state $\mathrm{U}_{2}$ is thereby linearly stable. Such an observation together with the result at a large perturbation confirms that the observed instability is induced from a metastable regime.

The states $\mathrm{L}_{1}$ and $\mathrm{L}_{2}$ in Fig. 5 are subject to liquid jetting. The symbol $\mathrm{P}_{1}$ denotes a 5 -s liquid jetting, and for $\mathrm{P}_{2}$, continuous liquid jetting. As Fig. 7 reveals, after an initial drop in fin base temperature, reflecting the occurrence of wet spot on fin surface, the state $\mathrm{L}_{2}$ would recover its original state. However, at a less base temperature, as the state $\mathrm{L}_{1}$, the system could recover when subject to perturbation $\mathrm{P}_{1}$, but is unstable to perturbation $\mathrm{P}_{2}$ (whose final state is $\mathrm{L}_{1}^{\prime}$ ). Again, the state along LSS becomes less stable when its base temperature is lower.

The transition paths as located in Fig. 5 are not vertical lines as suggested in Numerical Simulation section. Such an observation is attributed to the fact that in real process the base temperature cannot keep constant; but is determined by both the fin body and the heating assembly. Nevertheless, the basic characteristics of the experimental results correlate well with the theoretical findings.

\subsection{Implications to fin boiling applications}

The existence of metastable regimes in fin boiling has vital effects on its design and operational philosophy. In bistable regime where two steady states coexist, as Lin and Lee [15] addressed, the relatively less stable steady state is termed as 'metastable'. When an external perturbation was imposed, like dry spot in nucleate boiling mode or a wet spot in film boiling mode, the metastable steady state may lose its stability and induce transition. In fin boiling this means the marked reduction of heat flux and sharp increase in surface temperature, thereby leading to device failure. A proper boiling fin design should take not only the absolutely unstable points, such as point $\mathrm{A}$ and $\mathrm{C}$ in Fig. 5, but also the stable and metastable regimes into consideration.

Furthermore, the present results strongly suggest that the metastable and stable regimes depend upon the way the fin was heated, which correlates with the case on a flat plate heater [18]. Further works are required to explore the relationship between heating methods, heater configurations, and the metastability of fin boiling processes.

\section{Conclusions}

This work has, for the first time, numerically and experimentally elucidated the existence of metastable regimes in fin boiling process. In the first part the numerical simulation was conducted for analyzing the response of the linearly stable steady states subject to large external perturbations. As the linearly stable steady state moves toward the critical points on the boiling curve by increasing base temperature, which is linearly unstable in nature, the capability of the steady state to tolerate perturbation becomes weaker. On the basis of these information the stable and metastable regimes could be subsequently identified. Experimental results with methanol boiling on a copper fin support these numerical findings. To operate at metastable boiling regime might induce (nonlinear) instability if the magnitude of external perturbation is large enough. Consequently, a proper boiling fin design should take into consideration both the stable and metastable regimes. 


\section{Acknowledgements}

The authors want to thank National Science Council, ROC for supporting this study.

\section{References}

[1] D.Q. Kern, A.D. Kraus, Extended Surface Heat Transfer, McGraw-Hill, NY, 1972.

[2] K.W. Haley, J.W. Westwater, Boiling heat transfer from a single fin to a boiling liquid, Chem. Eng. Sci. 20 (1965) 711.

[3] F.S. Lai, Y.Y. Hsu, Temperature distribution in a fin partially cooled by nucleate boiling, AIChE J. 13 (1967) 817-821.

[4] A.D. Kraus, Sixty-five years of extended surface technology, Appl. Mech. Rev. 41 (1988) 321-364.

[5] S.P. Liaw, R.H. Yeh, Fins with temperature dependent surface heat flux-I. Single heat transfer mode, Int. J. Heat Mass Transfer 37 (1994) 1509-1514.

[6] S.P. Liaw, R.H. Yeh, Fins with temperature dependent surface heat flux-II. Multi-boiling heat transfer, Int. J. Heat Mass Transfer 37 (1994) 1517-1524.

[7] H.C. Unal, Determination of the temperature distribution in an extended surface with a nonuniform heat transfer coefficient, Int. J. Heat Mass Transfer 28 (1985) 2279-2284.

[8] A.K. Sen, S. Trinh, An exact solution for the rate of heat transfer from a rectangular fin governed by power law-type temperature dependence, ASME J. Heat Transfer 108 (1986) 457-459 .
[9] W.W. Lin, D.J. Lee, Boiling on a straight pin fin, AIChE J. 42 (1996) 2721-2728.

[10] W.W. Lin, D.J. Lee, Experimental study on multi-mode methanol boiling on a straight pin fin, AIChE J. 45 (1999) 1147-1152.

[11] W.W. Lin, J.C. Yang, D.J. Lee, Boiling on a conical spine, Expr. Heat Transfer 12 (1999) 175-191.

[12] Z.W. Liu, W.W. Lin, D.J. Lee, Boiling of FC-72 on straight pin fin, Int. Comm. Heat Mass Transfer 26 (1999) 311-318.

[13] F. Tachibana, M. Akiyama, H. Kawamura, Nonhydrodynamic aspects of pool boiling burnout, J. Nucl. Sci. Tech. 4 (1967) 121-130.

[14] D.J. Lee, S.M. Lu, Two-mode boiling on a horizontal heating wire, AIChE J. 38 (1992) 1115-1128.

[15] W.W. Lin, D.J. Lee, Relative stability between nucleate and film boiling of flow boiling of methanol, ASME J. Heat Transfer 119 (1997) 326-331.

[16] W.W. Lin, D.J. Lee, Methanol flow boiling over a nonuniformly heated flat plate, J. Chin. Inst. Chem. Eng. 29 (1998) 27-32.

[17] W.W. Lin, J.C. Yang, D.J. Lee, Relative stability of boiling over nonuniformly heated surface: effects of heater orientation, ASME J. Heat Transfer 121 (1999) 484 486.

[18] W.W. Lin, J.C. Yang, D.J. Lee, Boiling stability characteristics of methanol flowing over a nonuniformly heated surface, Int. J. Heat Mass Transfer 41 (1998) 4009-4023.

[19] W.W. Lin, J.C. Yang, D.J. Lee, Boiling on a straight pin fin with an insulated or uninsulated tip, J. Enhanced Heat Transfer 5 (1998) 127-138. 